\title{
Dysregulated miR-671-5p / CDR1-AS / CDR1 / VSNL1 axis is involved in glioblastoma multiforme
}

\author{
Davide Barbagallo", ${ }^{1, \pi}$ Angelo Condorellii, ${ }^{1, \pi}$ Marco Ragusa ${ }^{1, \pi}$, Loredana Salito ${ }^{1}$, \\ Mariangela Sammito ${ }^{1}$, Barbara Banelli ${ }^{2}$, Rosario Caltabiano ${ }^{3}$, Giuseppe Barbagallo ${ }^{3}$, \\ Agata Zappalà ${ }^{4}$, Rosalia Battaglia ${ }^{1}$, Matilde Cirnigliaro ${ }^{1}$, Salvatore Lanzafame ${ }^{3}$, \\ Enrico Vasquez ${ }^{3}$, Rosalba Parenti ${ }^{4}$, Federico Cicirata ${ }^{4}$, Cinzia Di Pietro ${ }^{1, *}$, Massimo \\ Romani ${ }^{2, *}$, Michele Purrello',* \\ ${ }^{1}$ Dipartimento di Scienze Biomediche e Biotecnologiche, Sezione di Biologia e Genetica G Sichel, Unità di BioMedicina \\ Molecolare, Genomica e dei Sistemi Complessi, Università di Catania, Catania, Italy, EU \\ ${ }^{2}$ UOS Epigenetica dei Tumori, IRCCS A.O.U. San Martino-IST, Genova, Italy, EU \\ ${ }^{3}$ Dipartimento di Scienze Mediche, Chirurgiche e Tecnologie Avanzate G.F. Ingrassia, Università di Catania, Catania, Italy, EU \\ ${ }^{4}$ Dipartimento di Scienze Biomediche e Biotecnologiche, Sezione di Fisiologia, Università di Catania, Catania, Italy, EU \\ "These authors contributed equally to this work \\ *Senior Corresponding Authors
}

Correspondence to: Michele Purrello, e-mail: purrello@unict.it

Keywords: glioblastoma multiforme (GBM), non coding RNAs (ncRNAs), microRNAs (miRNAs), circular RNAs (circRNAs), cell networks

Received: May 25, 2015

Accepted: November 14, 2015

Published: December 15, 2015

\section{ABSTRACT}

MiR-671-5p is encoded by a gene localized at 7q36.1, a region amplified in human glioblastoma multiforme (GBM), the most malignant brain cancer. To investigate whether expression of miR-671-5p were altered in GBM, we analyzed biopsies from a cohort of forty-five GBM patients and from five GBM cell lines. Our data show significant overexpression of miR-671-5p in both biopsies and cell lines. By exploiting specific miRNA mimics and inhibitors, we demonstrated that miR-671-5p overexpression significantly increases migration and to a less extent proliferation rates of GBM cells. Through a combined in silico and in vitro approach, we identified CDR1-AS, CDR1, VSNL1 as downstream miR-671-5p targets in GBM. Expression of these genes significantly decreased both in GBM biopsies and cell lines and negatively correlated with that of miR-671-5p. Based on our data, we propose that the axis miR671-5p / CDR1-AS / CDR1 / VSNL1 is functionally altered in GBM cells and is involved in the modification of their biopathological profile.

\section{INTRODUCTION}

According to the World Health Organization (WHO), gliomas are grouped into 4 histological grades (Grade I to IV) that are defined by increasing degree of anaplasia, undifferentiation, aggressiveness [1,2]. GBM is the most prevalent and aggressive cancer originating in the central nervous system, mainly in the brain [3]. Its prognosis is very poor, as GBM patients have a median overall survival of only 14 months. Overall age-adjusted incidence rates for all gliomas (ICD-O-3 morphology codes 9380-9480), normalized to the national population of each respective study, range from 4.67 to $5.73 / 100000$ persons. Ageadjusted incidence of GBM (ICD-O-3 morphology codes
9440-9442, WHO grade IV) ranges from 0.59 to 3.69 / 100000 persons [3]. Data on the most common somatic mutations and corresponding pathways have demonstrated a very high molecular heterogeneity of GBM tumors [4 6]. MicroRNAs (miRNAs) are known to play an important role in GBM pathogenesis and progression [7 - 9]. A recently discovered class of non-coding RNAs, known as circular RNAs (circRNAs), has been established as a new post-transcriptional gene expression regulatory layer $[10,11]$. CircRNAs are covalently closed noncoding RNAs, which regulate gene expression by acting as miRNA sponges, reducing their availability within the cell [11]. Cerebellar Degeneration-Related Protein 1 (CDR1) antisense (CDR1-AS, a miR-7 sponge also known 
as ciRS-7) is the only circRNA known to be targeted and degraded by a microRNA (miR-671-5p) [11]. Similar to its sense counterpart CDR1, it is highly expressed in the brain. It positively controls the expression of CDR1 mRNA, probably by stabilizing it through direct interaction [12]. It also functions as miR-7 sponge by sequestering it through multiple binding sites $[11,12]$. This molecular system is regulated by miR-671-5p, which under appropriate conditions leads to Argonaute RISC catalytic component 2 (AGO2) - mediated degradation of CDR1-AS [12]. The human gene encoding miR-671 maps at 7q36.1, a genomic region frequently amplified in GBM [13]. We sought to verify the involvement of miR-671 in GBM pathogenesis by applying the gene candidate approach. By exploiting specific miRNA mimics and inhibitors, we demonstrate here that its overexpression in GBM biopsies and cell lines contributes to increase migration and proliferation rates of GBM cells. Based on our data, we propose that the axis miR-671-5p / CDR1-AS / CDR1 / Visinin-like 1 (VSNL1) is functionally altered in GBM and suggest that miR-671$5 \mathrm{p}$ is a novel oncomiR in GBM.

\section{RESULTS}

\section{MiR-671-5p and miR-671-3p expression in GBM biopsies}

MiR-671-5p resulted significantly overexpressed in GBM biopsies compared to all reference tissues (average fold change $=13$-fold, $p$-value $<0.001$, Student's $t$-test). MiR-671-3p expression did not significantly differ compared to controls. We also observed a significant overexpression of miR-21 (average fold change $=13.3$ fold, $p$-value $<0.001$, Student's $t$-test) and a significant underexpression of miR-7 (average fold change $=-7.2$ fold, $p$-value $<0.001$, Student's $t$-test) in the same biopsies (Figure 1A).

\section{MiR-671-5p expression in GBM cell lines}

Mir-671-5p resulted more than twofold overexpressed in A172, CAS-1, DBTRG, SNB-19 and U-87 MG GBM cells compared to whole brain, astrocytes and the neuroblastoma cell line SK-N-BE (Figure 1B). Three out of the five GBM cell lines (A172, CAS-1, DBTRG) showed more than twofold miR-671-5p overexpression also respect to other two tumor cell lines (A375, HCT116) (Figure 1B). All GBM cell lines showed under - and overexpression of miR-7 and miR-21 respectively, compared to whole brain, as reported by literature (Figure 1B).

\section{CDR1-AS, CDR1, CHPF2, VSNL1 expression in GBM biopsies}

We identified 46 validated and 61 predicted targets of miR-671-5p (see Supplementary Tables 1 and 2): among them, we selected CDR1-AS, CHPF2 and VSNL1 for further analysis. CDR1-AS is a validated miR-671-5p target with intriguing gene expression regulatory features (see Introduction on circRNAs). CHPF2 is the host gene of miR-671-5p and there is some experimental evidence that is targeted by the same miRNA. Among the top 15 predicted targets (ordered by increasing mirSVR score), VSNL1 is a known tumor-suppressor gene regulating cell migration in several cancer types. We added CDR1 as further putative miR-671-5p target because its expression is known to be positively regulated by CDR1-AS (see Introduction and Discussion). Expression of the selected putative targets was analyzed in GBM biopsies and compared to normal brain parenchyma. We observed: (1) downregulation of CDR1 (average fold change = -2.84-fold; $p$-value $=0.028$, Student's $t$-test) and CDR 1 AS (average fold change $=-3.51$-fold, $p$-value $=0.008$, Student's $t$-test); (2) upregulation of CHPF2 (average fold change $>100$-fold, $p$-value $<0.001$, Student's $t$-test); downregulation of VSNL1 (average fold change $=-2.1$ fold, $p$-value $=0.107$, Student's $t$-test), statistically not significant (Figure 2A). CDR1 appeared significantly less expressed in males compared to females within our cohort ( $p=0.027$, Student's $t$-test). A negative correlation (statistically not significant) was observed between tumor size and CDR1 expression $(r=-0.24, p=0.094$, Spearman Rank-Order Correlation test). We did not observe any other correlation between the expression of miR-671-5p or its targets and the clinical features of our GBM cohort.

\section{CDR1-AS, CDR1, CHPF2, VSNL1 expression in GBM cell lines}

CDR1-AS and CDR1 resulted on average downregulated in GBM cell lines with respect to astrocytes and other cancer cell lines, with the only exception of HCT 116; CAS-1 showed the most impressive downregulation of CDR1-AS and CDR1. VSNL1 downregulation was common to all GBM cell lines and, on average, more pronounced with respect to other cancer cell lines, with the only exception of SN-K-BE. CHPF2 was overexpressed more than twofold in all GBM cell lines: similar to miR$671-5 p$, its overexpression appeared more pronounced in GBM cell lines than in other tissues (Figure 2B). Data on VSNL1 underexpression and CHPF2 overexpression in GBM cell lines were confirmed also at protein level, by using normal cerebral cortex as control tissue (Figure 2C).

\section{Negative correlation between expression of miR- 671-5p and of CDR1-AS, CDR1 and VSNL1 in GBM biopsies and cell lines}

Expression of miR-671-5p negatively correlated with that of CDR1-AS, CDR1, VSNL1 $(r=-0.56,-0.57$, $-0.32, p=1.33 \mathrm{e}-05,1.91 \mathrm{e}-05,0.021$, respectively; $n=54$, 
51, 52, respectively, Spearman's Rank-Order Correlation test) (Figure 3). A highly positive correlation was detected between CDR1-AS and CDR1 expression ( $r=0.938$, $p=0, n=51$, Spearman's Rank-Order Correlation test) (Figure 3). The correlation between miR-671-5p and CHPF2 expression was not significant $(r=0.0077$, $p=0.957, n=51$, Spearman's Rank-Order Correlation test) (Figure 3). Levels of CDR1-AS, CDR1 and VSNL1 transcripts significantly decreased or increased in DBTRG,
SNB19 and U-87 MG following transfection with miR671-5p mimics or inhibitors, respectively (Figure 4).

\section{MiR-671-5p stimulates cell migration and proliferation in GBM DBTRG, SNB-19 and U-87 MG cell lines}

DBTRG, SNB-19 and U-87 MG transfected with miR-671-5p mimics significantly increased their

A
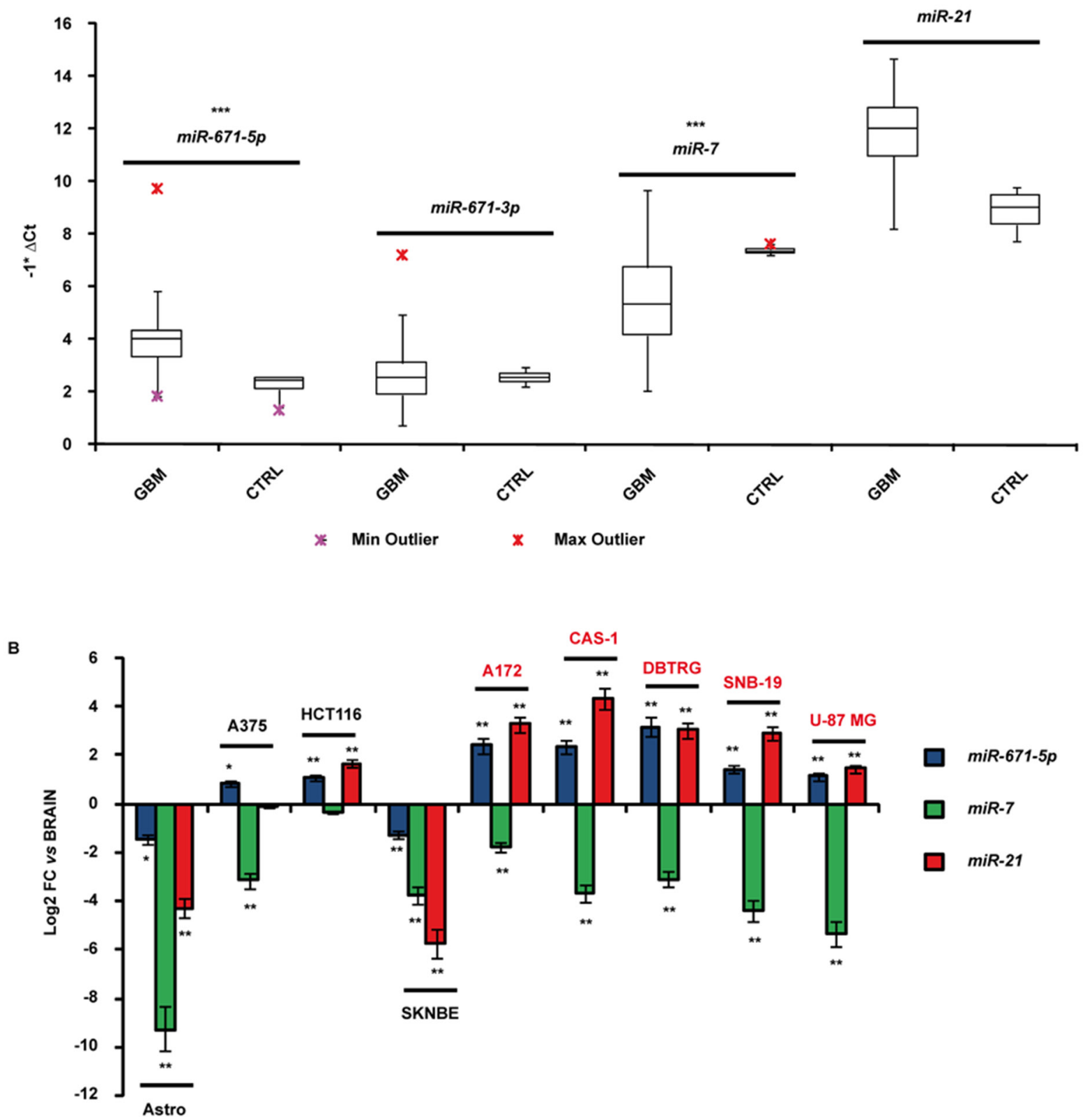

Figure 1: Expression of miR-671-5p, miR-671-3p, miR-21 and miR-7 in GBM biopsies A. and cell lines B. Expression values are reported as box plots with whiskers from minimum to maximum to represent $-1 * \Delta \mathrm{Ct}$, both in GBM biopsies as in controls (A), and as mean of fold change (FC) \pm Standard Deviation versus normal brain (B). miR-99a and miR-92a were used as reference genes in experiments on biopsies and cell lines, respectively. ${ }^{*} p$-value $<0.05, * * p$-value $<0.01$, ***p-value $<0.001$, Student's $t$-test $(n=3)$. 
A
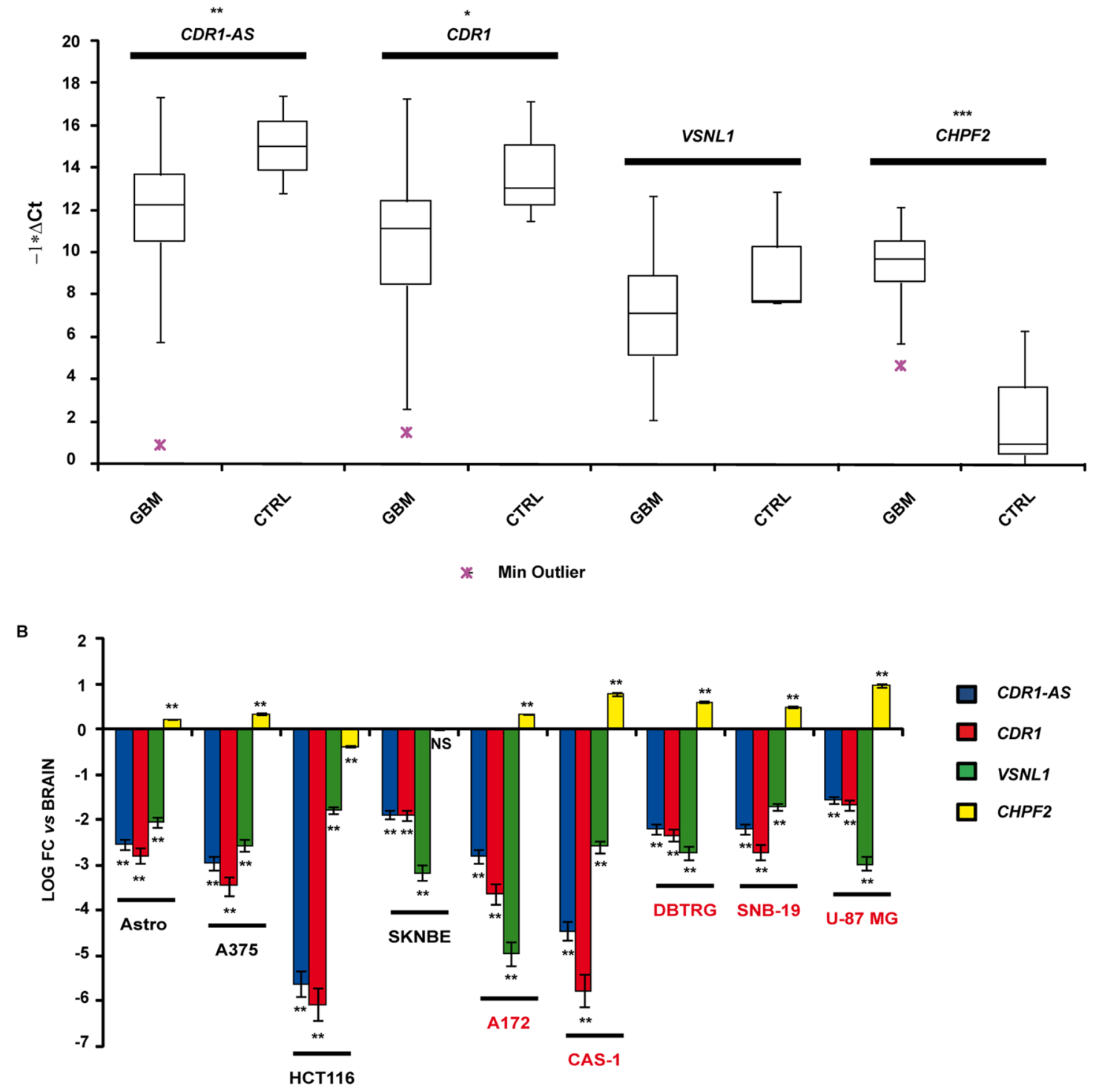

C

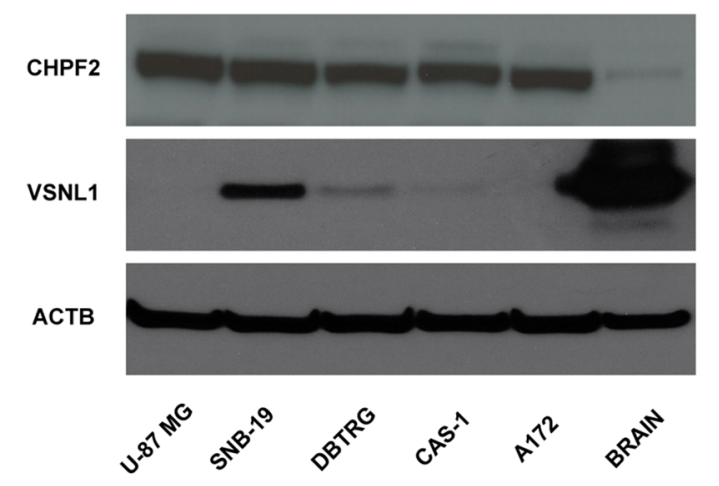

Figure 2: CDR1-AS, CDR1, VSNL1 and CHPF2 expression in GBM biopsies A. and cell lines B. Expression values are reported as box plots with whiskers from minimum to maximum to represent $-1 * \Delta \mathrm{Ct}$, both in GBM biopsies and controls (A), and as mean of fold change (FC) \pm Standard Deviation versus normal brain (B). Western blot of CHPF2 and VSNL1 in GBM cell lines and normal brain tissue C. ${ }^{*} p$-value $<0.05, * * p$-value $<0.01, * * * p$-value $<0.001$, Student's $t$-test $(n=3)$. 
migration rate of $15 \%, 32 \%$ and $15 \%$, respectively, $24 \mathrm{~h}$ after scratching, as compared to matched scrambletransfected cells (Figure 5). DBTRG cells transfected with miR-671-5p mimics significantly increased their viability $(16 \%)$ at $24 \mathrm{~h}$ after transfection; on the other hand, the same cells treated with miR-671-5p inhibitors

A

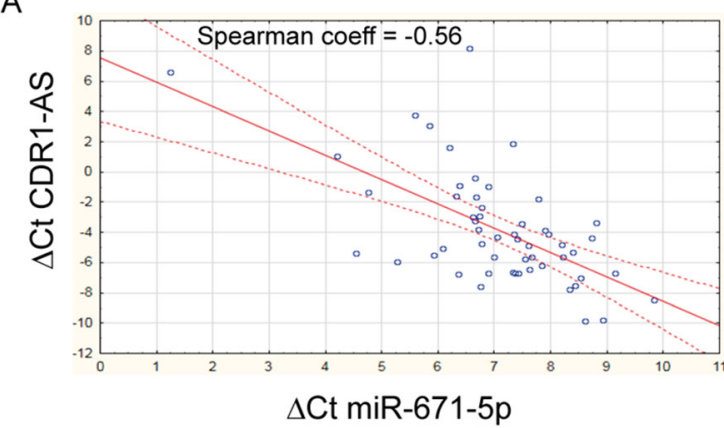

C

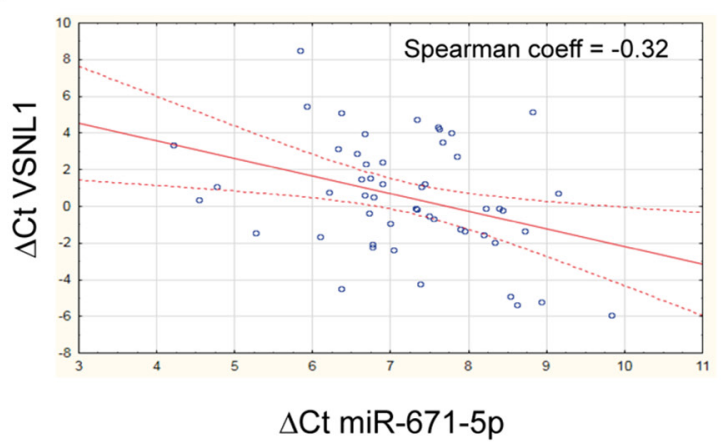

$E$

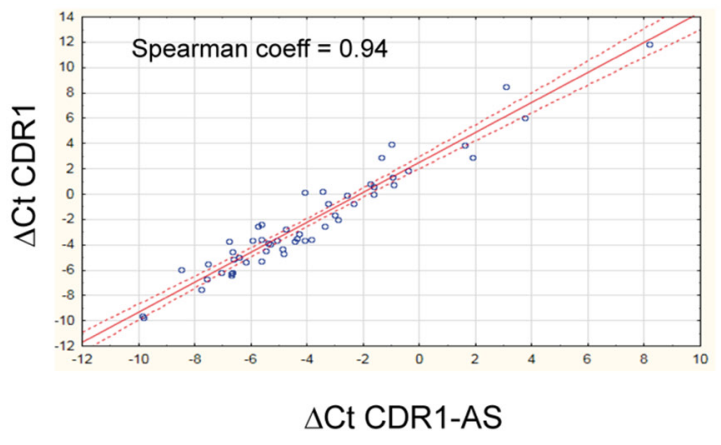

significantly decreased their viability (12\%) $72 \mathrm{~h}$ after transfection. SNB-19 showed a significant increase of viability (9\%) $48 \mathrm{~h}$ after transfection with miR-671-5p mimics and a significant reduction of viability (7\%) 72 $\mathrm{h}$ after transfection with miR-671-5p inhibitors. U-87 MG significantly increased their viability $(5 \%) 48 \mathrm{~h}$ after

B

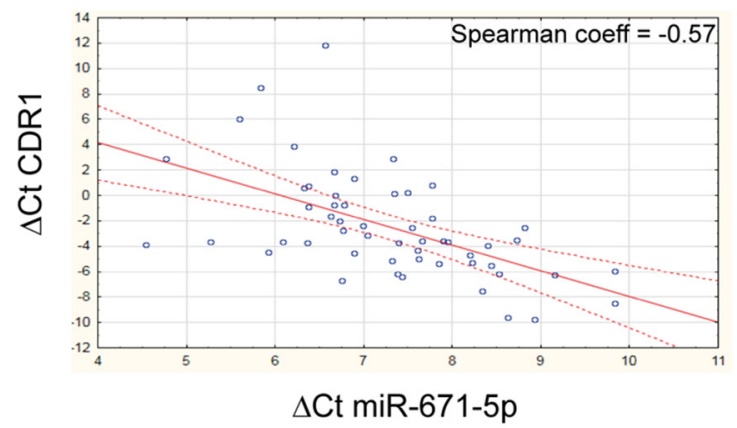

D

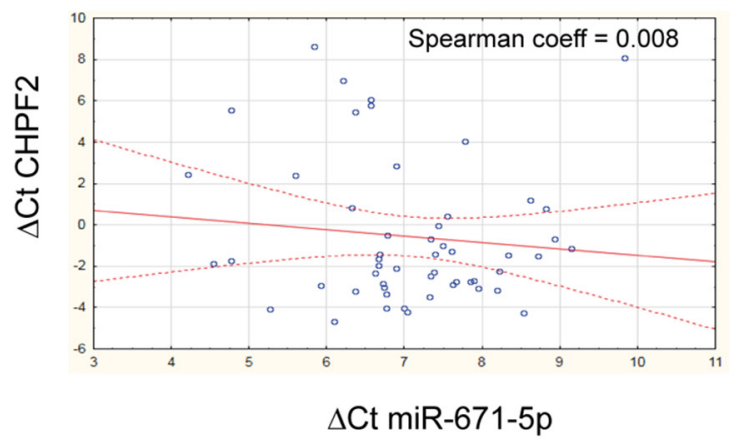

Figure 3: Scatter plots showing correlation between expression of miR-671-5p and its targets. Spearman's nonparametric rank correlation coefficients were calculated using $\Delta \mathrm{Ct}$ values of miR-671-5p and its targets CDR1-AS A. CDR1 B. VSNL1 C. CHPF2 D. and $\Delta \mathrm{Ct}$ values of CDR1-AS and CDR1 E. See text for details. 
A
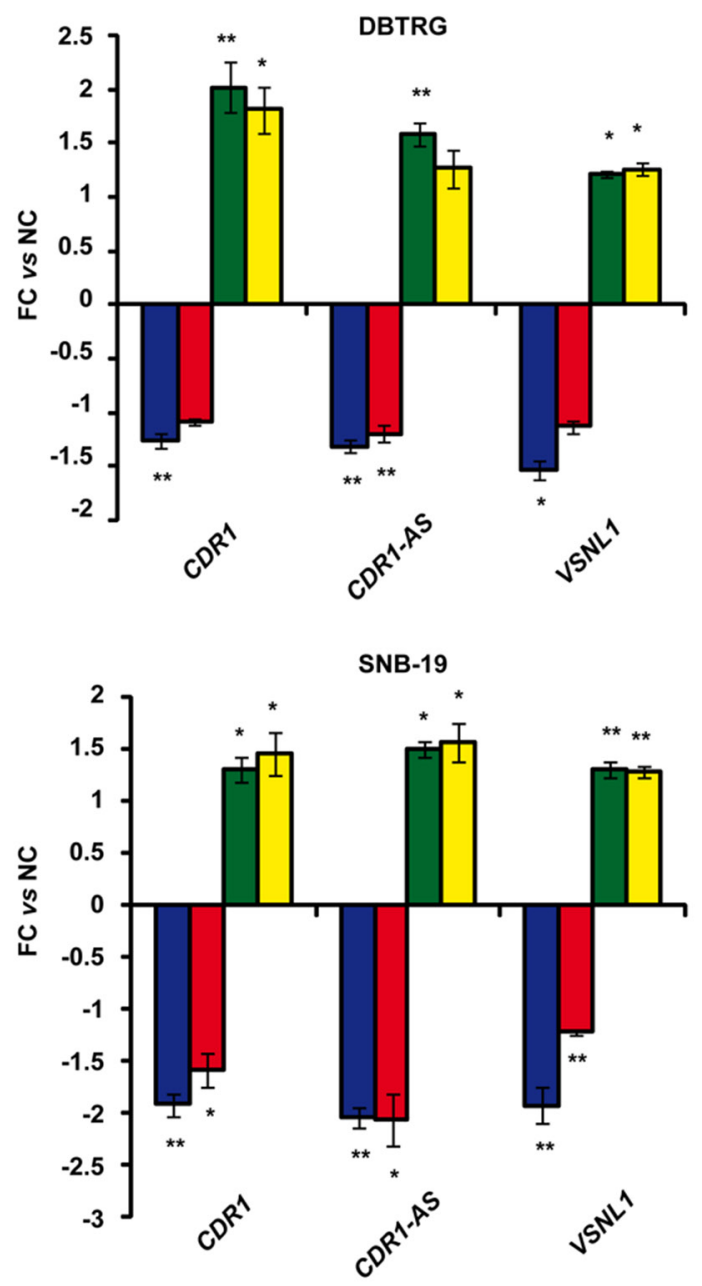

C

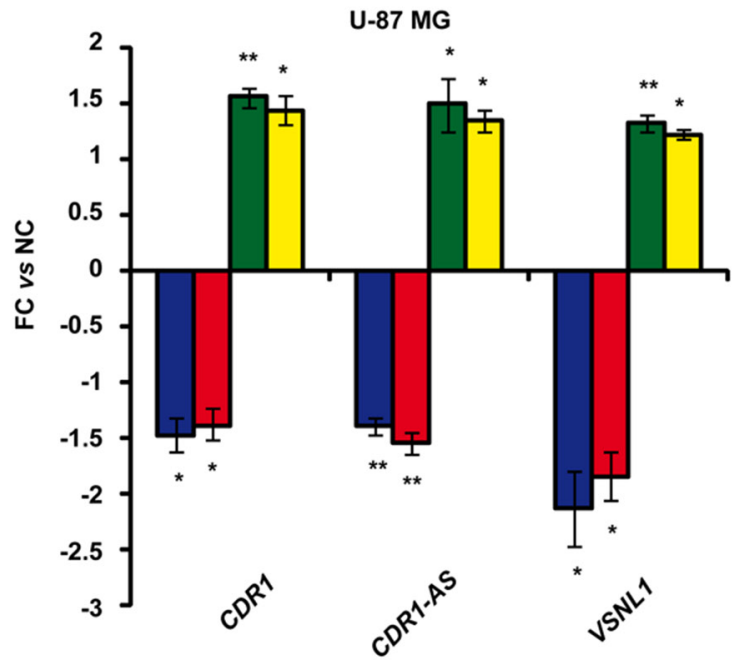

miR-671-5p Mim 24h

miR-671-5p Mim 48h

$m i R-671-5 p$ Inh 24h

$m i R-671-5 p$ Inh $48 \mathrm{~h}$
miR-671-5p Mim 24h

miR-671-5p Mim 48h

$m i R-671-5 p \operatorname{lnh} 24 \mathrm{~h}$

$m i R-671-5 p$ Inh $48 \mathrm{~h}$ $m i R-671-5 p$ Mim 24h

$m i R-671-5 p \operatorname{Mim} 48 \mathrm{~h}$

miR-671-5p Inh 24h

$m i R-671-5 p$ Inh $48 \mathrm{~h}$

Figure 4: Expression of CDR1-AS, CDR1, VSNL1 in DBTRG, SNB-19, U-87 MG cell lines after transfection with miR-671-5p mimics (miR-671-5p Mim) or inhibitors (miR-671-5p Inh). Expression values are reported as mean of fold change $(\mathrm{FC}) \pm$ Standard Deviation versus scramble molecules (NC) - transfected cells. * $p$-value $<0.05$; ** $p$-value $<0.01$, Student's $t$-test $(n=3)$. 
A
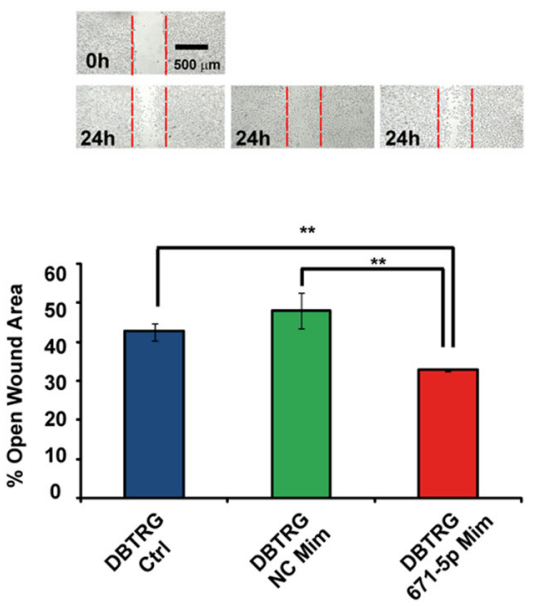
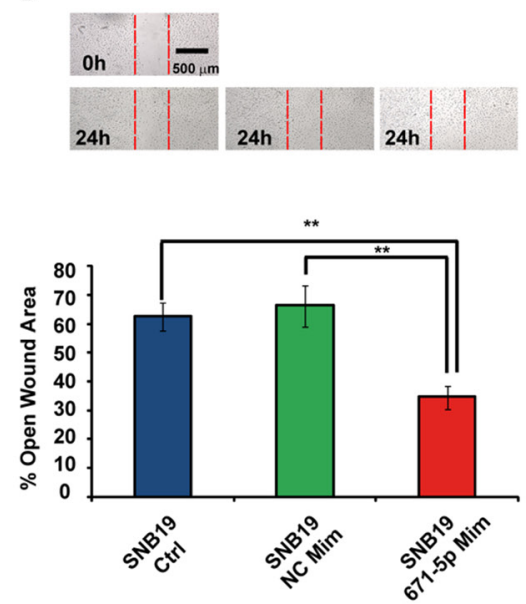

C

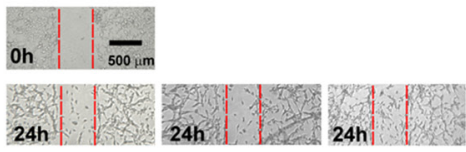

Figure 5: Involvement of miR-671-5p in DBTRG, SNB-19, U-87 MG migration. MiR-671-5p mimics stimulate cell migration with respect to scramble molecules (NC Mim) - transfected DBTRG A. SNB-19 B. U-87 MG C. cells in a wound-healing assay. Data are represented as mean percentage \pm Standard Deviation of open wound area of three independent biological replicates, $24 \mathrm{~h}$ after scratch. Magnification: $\times 8$. Results are representative of at least three randomly selected areas, assayed for each well. ${ }^{*} p$-value $<0.05$; $* * p$-value $<0.01$, Student's $t$-test.

transfection with miR-671-5p mimics, while significantly decreased viability $(19 \%) 48 \mathrm{~h}$ after transfection with miR-671-5p inhibitors (Figure 6).

\section{Network generation and analysis}

Starting from CDR1 and VSNL1 as input nodes, GeneMANIA algorithm generated a network of 240 nodes and 3935 edges. Edges included validated physical (protein-protein interaction) and genetic interactions, in addition to predicted microRNA-target and transcriptional factor-target interactions. CentiScaPe plug-in v.2.1 applied to the whole network revealed 16 nodes, whose centrality values were equal or higher than thresholds for all selected parameters: Betweenness, Bridging, Closeness, Degree. VSNL1 turned out to be a hub node. Literature mining showed that 15 among the most central nodes in the network are causally involved in the pathogenesis of GBM (specifically, in survival, proliferation, migration and invasion of GBM cells) (Table 1). Functional enrichment analysis performed by ClueGO v2.1.5 on the whole network revealed a statistically significant enrichment in terms like glioma KEGG pathway, central nervous system development, gliogenesis, negative regulation of neuronal apoptosis (see Supplementary Figure 1). The union of the subnetworks of CDR1 and VSNL1 plus their first neighbor interactants generated a new network consisting of 71 nodes and 357 edges (Figure 7). Within this more restricted network, we pinpointed contactin 6 (CNTN6) as the only node that links CDR1 and VSNL1 by a genetic interaction, in addition to 7 miRNAs (miR-23a, miR-23b, miR-124a, miR-196a, miR-196b, miR-369-3p, miR-381) controlling different nodes.

\section{DISCUSSION}

MiR-671-5p marked overexpression in GBM and its downstream effects on GBM cells migration and viability strongly suggest that this miRNA is a new oncomiR in GBM. There also are other evidences in literature that support this proposal [14-16]. Based on our in silico and experimental data, we further propose that CDR1-AS and VSNL1 are direct miR-671-5p targets, downexpressed in GBM (see Supplementary Figure 2). We also propose that CDR1 is an indirect target of miR-671-5p in the same biological context: its downregulation could be consequent to miR-671-5p-mediated degradation of CDR1-AS, as also suggested by literature [12]. CDR1AS belongs to the recently discovered class of circRNAs: among them, it is the only one known to be degraded by a miRNA: miR-671-5p [12]. CircRNAs expression appears to be frequently altered in cancer [17]. CDR1AS has been proposed as candidate gene in Alzheimer's Disease [18] and Diabetes Mellitus [19], but no data have been published on its potential involvement in GBM. Its downregulation, mediated by increased expression of miR-671-5p and associated to downregulation of CDR1, paves the way to the study of new pathways involved in GBM pathogenesis. CDR1 downregulation (likely linked to miR-671-5p-dependent CDR1-AS degradation) may indeed contribute to loss of differentiation of neural cells, leading to neoplastic transformation [20]. On the other hand, VSNL1 is known to inhibit cell migration and behaves as tumour suppressor in several cancer types [21 - 23]: its downregulation may lead to increased migration rate in GBM cells. Integration of CDR1 and VSNL1 within a physical and genetic interaction network 

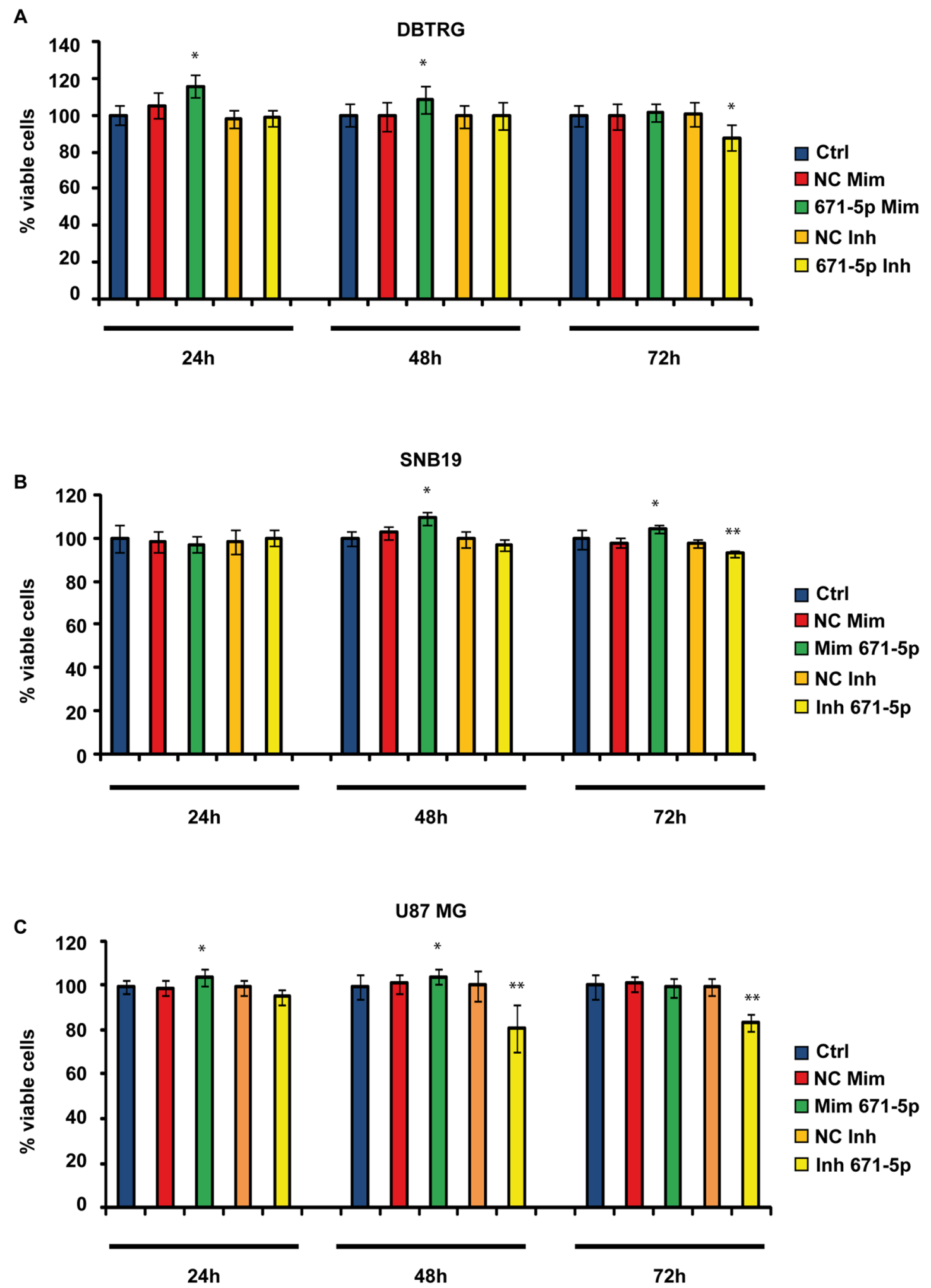

Figure 6: MTT assay in GBM cell lines DBTRG (A), SNB-19 (B), U-87 MG (C). Data are reported as percentages of viable cells, relative to controls. Experiments were performed as six biological replicates. ${ }^{*} P<0.05, * * P<0.01$, Student's $t$-test. Ctrl (not transfected cells); NC Mim (Cells transfected with scramble molecules of miRNA mimics); 671-5p Mim (Cells transfected with miR-671-5p mimics); NC Inh (Cells transfected with scramble molecules of miRNA inhibitors); 671-5p Inh (Cells transfected with miR-671-5p inhibitors). 
Table 1: Hub nodes within miR-671-5p targets' network

\begin{tabular}{|c|c|c|c|c|c|}
\hline Node name & Betweenness & Bridging & Closeness & Degree & GBM reference \\
\hline $\mathrm{BNC2}$ & 349.06 & 4.89 & 0.0023 & 48 & PMID: 24607568 \\
\hline CAGGTA_V\$AREB6_01 [ZEB1] & 4973.51 & 5.29 & 0.0029 & 135 & PMID: 23818228 \\
\hline CTTTGA_V\$LEF1_Q2 [LEF1] & 1576.5 & 4.76 & 0.0025 & 83 & PMID: 25128061 \\
\hline CXCR4 & 249.93 & 5.46 & 0.0022 & 36 & PMID: 12388552 \\
\hline ETV1 & 250.47 & 5.3 & 0.0022 & 39 & PMID: 19148472 \\
\hline GAB2 & 296.42 & 4.81 & 0.0023 & 46 & PMID: 23231021 \\
\hline GRID2 & 836.94 & 4.94 & 0.0025 & 73 & PMID: 19011622 \\
\hline JAG1 & 583.17 & 4.96 & 0.0024 & 64 & PMID: 22296176 \\
\hline LMO3 & 598.75 & 4.98 & 0.0024 & 62 & PMID: 25829251 \\
\hline NR1D1 & 269.82 & 4.84 & 0.0023 & 45 & PMID: 19011622 \\
\hline SRPK2 & 293.81 & 5.59 & 0.0022 & 36 & PMID: 19011622 \\
\hline $\begin{array}{l}\text { TGGAAA_V\$NFAT_Q4_01 } \\
\text { [NFATC1, NFATC2] }\end{array}$ & 3691.69 & 4.73 & 0.0028 & 129 & PMID: 23762456 \\
\hline V\$FOXM1_01 [FOXM1] & 456.43 & 4.98 & 0.0022 & 46 & PMID: 22977194 \\
\hline V\$GATA1_05 [GATA1] & 1031.95 & 4.76 & 0.0024 & 65 & $\begin{array}{l}\text { http://digitalcommons.wayne } \\
\text {.edu/oa_dissertations/87 }\end{array}$ \\
\hline V\$GATA3_01 [GATA3] & 555.79 & 5.02 & 0.0022 & 50 & $\mathrm{~N} / \mathrm{A}$ \\
\hline VSNL1 & 1411.43 & 8.66 & 0.0024 & 63 & PMID: 20525252 \\
\hline Threshold & 221.74 & 4.71 & 0.0021 & 32.79 & \\
\hline
\end{tabular}

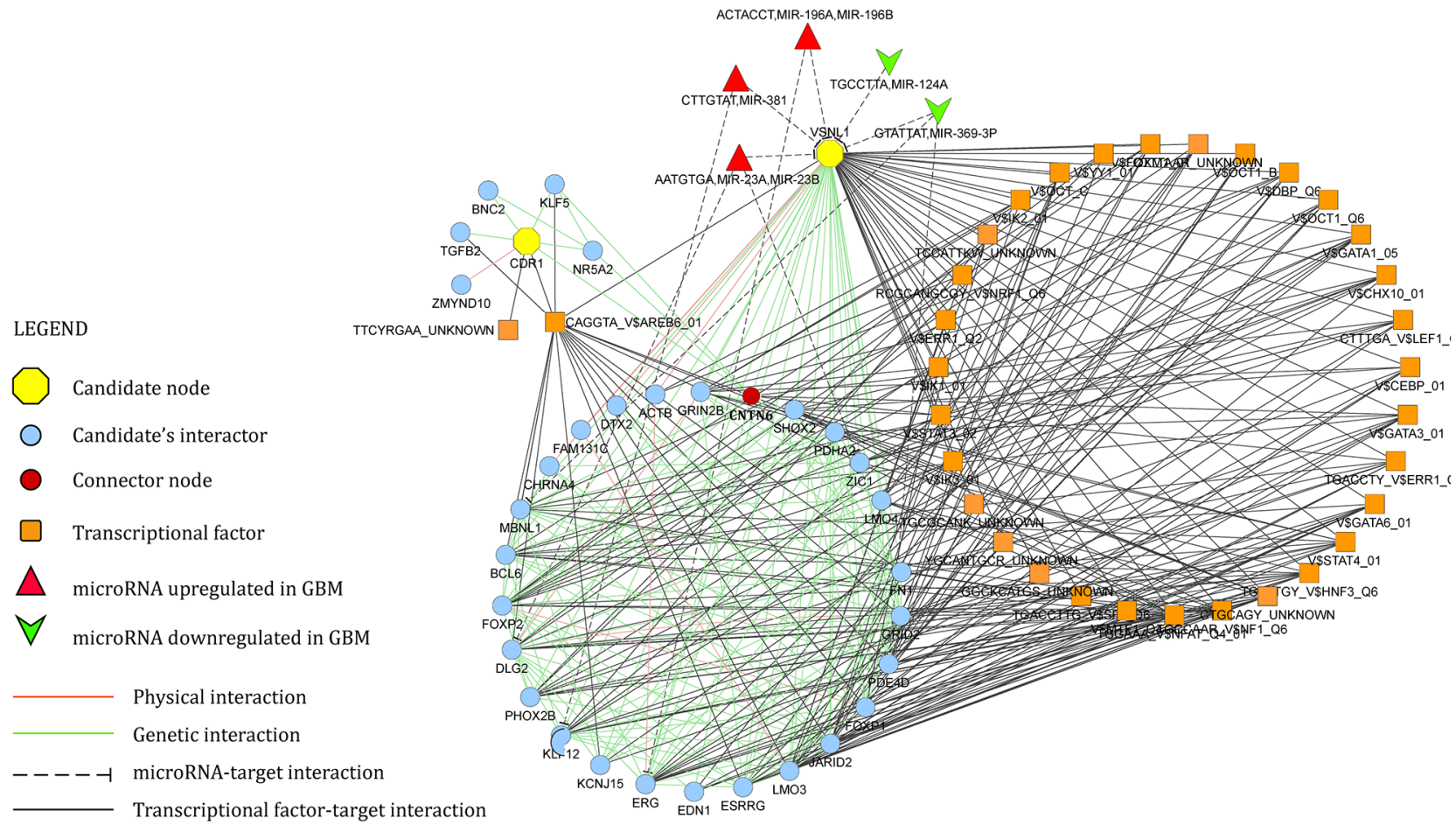

Figure 7: Network of CDR1 and VSNL1 (candidate nodes) plus their first neighbor interactants. See legend within the figure and text for further details. 
strongly supports our hypothesis on their involvement in GBM: the network is centered on 16 nodes (hub nodes), 15 of which are involved in glioma cell pathways and in biological functions as central nervous system development, gliogenesis, negative regulation of neuron apoptosis (see Table 1 and Supplementary Figure 1). VSNL1, a hub node within the network, is predicted to be targeted by 7 miRNAs, 5 of which are oncomiRNAs highly expressed in GBM (miR-23a, miR-23b, miR-196a, miR-196b, miR-381) (Figure 7) [24-28]. This circuitry may be upstream fostered by SOX2 (Sry-related box2) in GBM cells: this transcription factor is known to promote malignancy in GBM and to positively control the expression of miR-671-5p [29, 30]. Interestingly, literature mining and target analysis reveal that both miR-671-5p and SOX-2 may contribute to inhibiting the expression of common targets, like Solute Carrier Family 7 (Cationic Amino Acid Transporter, y+ System) member 1 (SLC7A1) or Fibronectin type III domain-containing 5 (FNDC5) [29, $30]$. FNDC5 was earlier described as involved in neural cell differentiation [31].

\section{MATERIALS AND METHODS}

\section{Cell cultures and transfection}

A172, CAS-1, DBTRG, HCT-116, SK-N-BE, SNB19, U-87 MG cell lines were obtained from the Interlab Cell Line Collection (ICLC), an International Repository Authority within IST (IRCCS Azienda Ospedaliera Universitaria San Martino- IST - Istituto Nazionale per la Ricerca sul Cancro, Genova, Italy, EU). A375 cell line was purchased from American Type Culture Collection (ATCC). HCT-116 and SK-N-BE cells were grown as previously reported [32, 33]. Further details on culture conditions of other cell lines are provided in Supplementary Methods. Characterization and validation of cell lines were performed by the cell banks. Cell lines were verified to be mycoplasma-free by Hoechst staining and PCR (TIB Molbiol) and by MycoTect (Gibco BRL). Species verification was performed by isoenzyme analysis (AuthentiKit TM System, Innovative Chemistry). Further, profiling of multiplex Short Tandem Repeats was performed to verify identity and uniqueness of cell lines. After receiving the cells, an aliquot was immediately frozen, whereas another was cultured up to the $10^{\text {th }}$ passage to perform the experiments. MiR-671-5p mimics and inhibitors (anti-miR-671-5p) were purchased from Lifetechnologies $^{\mathrm{TM}}$ (Carlsbad, CA, USA). For transfection experiments, cells were seeded at a density of 50000 per well in a 24-well plate and transfected using siPORT ${ }^{\mathrm{TM}}$ NeoFX ${ }^{\mathrm{TM}}$ (LifeTechnologies ${ }^{\mathrm{TM}}$ ). Final concentrations of miR-671-5p mimics and inhibitors were determined according to the best knock-in or knock-down efficiency, for each transfected cell line (see Supplementary Figure 3).

\section{Cohort of GBM patients}

Forty-five paraffin-embedded biopsy samples from GBM patients (GBM: WHO Grade IV) were obtained by the Pathology Laboratory of the Department of Advanced Technologies in Medical and Surgical Sciences G.F. Ingrassia, University of Catania, Catania, Italy, EU. The study was approved by the local ethical committee. Cases were selected on the basis of availability of adequate tumor tissue: haematoxylin- and eosin- (H\&E) stained slides were reviewed and a concordant diagnosis was performed by trained pathologists, based on WHO 2007 tumor classification [1]. To avoid contamination with nontumor tissue, the tumor areas were macrodissected with sterile disposable scalpels and then subjected to RNA isolation. Before extraction of total RNA from tumor tissue, each macrodissected section was stained with haematoxylin and eosin for the evaluation of purity of the biopsy. Employing this procedure, only tumor tissue was included and evaluated in the study. Demographic data, clinical and pathological parameters on GBM patients are reported in Supplementary Table 3. Three paraffinembedded control brain biopsy samples were obtained from the frontal cerebral area as non-neoplastic reference tissues. We avoided using control tissues adjacent to the tumor, since there is the possibility of undetected tumor cell infiltration. Data on EGFR amplification and protein expression were obtained by Fluorescence In Situ Hybridization (FISH) and immunohistochemistry (IHC) (see Supplementary Table 3) [34]. Data on O-6methylguanine-DNA methyltransferase (MGMT) methylation were obtained as previously reported (see Supplementary Table 3) [35]. Volumetric data on biopsies were retrieved through volumetric analysis of postoperative gadolinium (Gd)-enhanced magnetic resonance (MR) images, obtained within $48 \mathrm{~h}$ following surgery (see Supplementary Table 3) [35].

\section{RNA isolation and quantitative real-time RT-PCR}

Total RNA from formalin-fixed paraffin-embedded (FFPE) biopsies and from cell lines was purified using RecoverAll ${ }^{\mathrm{TM}}$ Total Nucleic Acid Isolation Kit (LifeTechnologies ${ }^{\mathrm{TM}}$ ) and TRIzol $^{\circledR}$ (LifeTechnologies ${ }^{\mathrm{TM}}$ ), respectively. Total whole Brain and Astrocytes RNA was from FirstChoice ${ }^{\circledR}$ Human Total RNA Survey Panel (Ambion ${ }^{\circledR}$, Austin, TX) and ScienCell Research Laboratories $^{\circledR}$ (San Diego, CA), respectively. RNA was quantified and treated with DNase I Amplification Grade (LifeTechnologies ${ }^{\mathrm{TM}}$ ). 30ng of DNase-treated RNA were reverse transcribed by using specific RT miRNA primers and amplified with specific TaqMan ${ }^{\circledR}$ MicroRNA assays (LifeTechnologies ${ }^{\mathrm{TM}}$ ). MiR-92a and miR-99a resulted the most stable miRNAs by applying GeNORM [36]: accordingly, they were used as reference miRNA genes (see Figure 1 legend). 50 ng of DNase-treated RNA from 
GBM biopsies were reverse transcribed and amplified with target-specific primers in a one-step quantitative real-time PCR reaction through Power SYBR ${ }^{\circledR}$ Green RNA-to- $\mathrm{C}_{\mathrm{T}}{ }^{\mathrm{TM}}$ 1-Step Kit (LifeTechnologies ${ }^{\mathrm{TM}}$ ). $1 \mu \mathrm{g}$ of DNase-treated RNA from cell lines was reverse transcribed through HighCapacity RNA-to-cDNA ${ }^{\mathrm{TM}}$ Kit (LifeTechnologies ${ }^{\mathrm{TM}}$ ) and $20 \mathrm{ng}$ of the resulting cDNAs were amplified with target-specific primers by $\mathrm{SYBR}^{\circledR}$ Green Master Mix (LifeTechnologies ${ }^{\mathrm{TM}}$ ). TBP was used as reference gene, as it resulted the most stable gene on applying GeNORM. Primer sequences are available upon request. Relative quantification of gene expression was calculated using the comparative $\mathrm{Ct}\left(2^{-\Delta \Delta \mathrm{Ct}}\right)$ method [37]. We considered differential all the expression values that were statistically significant different between control and test conditions, as previously described [38, 39].

\section{Western blot analysis}

Protein lysates from GBM cell lines were obtained as previously described [39]. Human brain cerebral cortex protein medley was purchased from Takara Clontech ${ }^{\circledR}$ (Mountain View, CA, USA). $40 \mu \mathrm{g}$ of total protein extract were loaded into Bolt ${ }^{\mathrm{TM}} 4-12 \%$ Bis-Tris Plus Gel (Lifetechnologies ${ }^{\mathrm{TM}}$ ) and blotted to nitrocellulose membranes by iBlot Dry Blotting System (Lifetechnologies ${ }^{\mathrm{TM}}$ ). Membranes were probed with polyclonal antibodies to CHPF2 (Sigma-Aldrich ${ }^{\circledR}$, Saint Louis, MO, USA) [tested at http://www.proteinatlas.org/ ENSG00000033100-CHPF2/antibody] and monoclonal

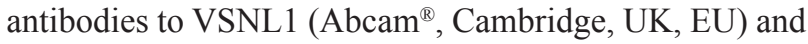
$\beta$-actin (Sigma-Aldrich ${ }^{\circledR}$ ) [38]. $\beta$-actin was used as loading control. Proteins were detected by using ECL ${ }^{\text {TM }}$ Western Blotting Detection Reagents (GE Healthcare ${ }^{\odot}$ ).

\section{miR-671-5p targets selection}

Validated targets of miR-671-5p were retrieved from miRTarbase, release 4.5 [40]. Top 200 predicted targets of miR-671-5p were obtained through TargetScanHuman online algorithm, release 6.2 [41]. The list of predicted targets was further filtered by removing genes: (i) whose expression did not negatively correlate with that of miR-671-5p; (ii) had a miRanda-mirSVR score $>-0.5$. Negative correlation among putative targets and miR671-5p was analyzed through miRGator v.3.0 (http:// mirgator.kobic.re.kr) [42]. MiRanda-mirSVR scores (available at http://www.microrna.org) indicate the probability that a miRNA $v s$ predicted target alignment lead to downregulation of the latter: more negative is the score, more stringent is the prediction [43]. Final selection of putative targets for downstream experimental analysis has been based on literature mining, following these criteria: (i) unknown involvement in GBM pathogenesis; (ii) tumor suppressor genes with known correlation with cancer, neuronal differentiation or cell migration. Tumor suppressor genes were retrieved within the list of predicted targets by Tumor Suppressor Gene Database (http:// bioinfo.mc.vanderbilt.edu/TSGene/).

\section{Wound-healing assay}

Cells were plated in 24-well plates and grown to $80 \%-90 \%$ confluence by using the same transfection protocol described above. A wound was created in the cell layer by using a pipette tip. Cells were washed twice with PBS to remove cell debris and floating cells and were refeeded with new serum-free medium for $24 \mathrm{~h}$. Wounds were subsequently observed under an inverted microscope (Leitz, Fluorvert FU): images covering the entire width of the wounds were captured on camera (Leica, DFC 495), at time zero and after $24 \mathrm{~h}$, by using an $8 \times$ objective. TScratch v. 1.0 software was used to calculate the percentage of open wound area for each condition. At least three randomly selected areas were assayed for each well. Results are shown as the mean \pm standard deviation of the percentage of open wound area of three biological replicates.

\section{MTT assay}

Cell viability was evaluated through MTT assay. Briefly, $10^{4}$ cells / well were reverse transfected with miR671-5p mimics or inhibitors or scramble molecules, seeded into 96 -well plates and incubated at $37^{\circ} \mathrm{C}$ for $24,48,72 \mathrm{~h}$. At the end of each time point, cells were incubated for $3 \mathrm{~h}$ with $5 \mathrm{mg} / \mathrm{ml}$ of MTT solution (serum-free medium was used as solvent). Washing with phosphate-buffered saline (PBS) was followed by addition of dimethyl sulfoxide (DMSO). The solution was gently shaken for 10', so that complete dissolution of formazan crystals was achieved. Absorbance was recorded at $550 \mathrm{~nm}$, using the microplate spectrophotometer system Multiscan Ascent ${ }^{\circledR}$ microplate reader (Thermo Fisher Scientific). Data were exported and analyzed through Excel. Final results are presented as the mean \pm standard deviation of the percentage of cell viability of six biological replicates.

\section{Network generation and analysis}

A network of physical and genetic interactions was generated by GeneMANIA plug-in $[44,45]$ in Cytoscape v3.2.0 [46]. Specifically, CDR1 and VSNL1 were given as input to GeneMANIA plug-in. Network centrality parameters Betweenness, Bridging centrality, Closeness and Degree were analyzed through CentiScaPe plugin v.2.1 [47]. All the nodes having network centrality parameter values above the mean (threshold) were considered hubs of the network. Functional enrichment analysis (Gene ontologies and KEGG pathways) among the nodes of the network was performed through the Cytoscape plug-in ClueGO v2.1.5 [48]. 


\section{Statistical analysis}

Comparison between two experimental groups was performed through Student's $t$-test. To compare three or more groups, we used one way ANOVA. Spearman RankOrder Correlation Coefficient was calculated to correlate gene expression values. $P$-values $<0.05$ were considered to be statistically significant.

\section{ACKNOWLEDGMENTS}

We thank Dr. J Kjems and his collaborators for sharing information on CDR1-AS, Dr. E Pricoco for samples preparation with microtome, Dr. F Certo for compiling the clinical register of GBM patients, Prof. V D'Agata, Dr. G D'Amico and Dr. G Maugeri for sharing microplate reader with us. We also acknowledge the technical collaboration of Mr. A Vasta and thank Dr. A Scacciante (CO.DI.SAN Spa) for his kind cooperation.

\section{CONFLICTS OF INTEREST} interest.

The authors disclose no potential conflicts of

\section{GRANT SUPPORT}

This work was supported by funds from MIUR (Italian Ministry for Instruction, University and Research) to MP and CDP. MR was supported by Fondazione Compagnia di San Paolo Torino and by the Italian Ministry of Health, Core Grant Ricerca Corrente to the IRCCS AOU San Martino-IST. BB was the recipient of the Young Investigators grant GR-2008-1143408 from the Italian Ministry of Health.

\section{REFERENCES}

1. Louis DN, Ohgaki H, Wiestler OD, Cavenee WK, Burger PC, Jouvet A, Scheithauer BW, Kleihues P. The 2007 WHO classification of tumours of the central nervous system. Acta Neuropathol. 2007; 114: 97-109.

2. Dolecek TA, Propp JM, Stroup NE, Kruchko C. CBTRUS statistical report: primary brain and central nervous system tumors diagnosed in the United States in 2005-2009. Neuro-Oncol. 2012; 14: v1-49.

3. Ostrom QT, Bauchet L, Davis FG, Deltour I, Fisher JL, Langer CE, Pekmezci M, Schwartzbaum JA, Turner MC, Walsh KM, Wrensch MR, Barnholtz-Sloan JS. The epidemiology of glioma in adults: a "state of the science" review. Neuro-Oncol. 2014; 16: 896-913.

4. Sturm D, Bender S, Jones DT, Lichter P, Grill J, Becher O, Hawkins C, Majewski J, Jones C, Costello JF, Iavarone A, Aldape K, Brennan $\mathrm{CW}$ et al. Paediatric and adult glioblastoma: multiform (epi)genomic culprits emerge. Nat Rev Cancer. 2014; 14: 92-107.

5. Patel AP, Tirosh I, Trombetta JJ, Shalek AK, Gillespie SM, Wakimoto H, Cahill DP, Nahed BV, Curry WT, Martuza RL, Louis DN, Rozenblatt-Rosen O, Suvà ML et al. Singlecell RNA-seq highlights intratumoral heterogeneity in primary glioblastoma. Science. 2014; 344: 1396-1401.

6. Brennan CW, Verhaak RG, McKenna A, Campos B, Noushmehr H, Salama SR, Zheng S, Chakravarty D, Sanborn JZ, Berman SH, Beroukhim R, Bernard B, Wu CJ et al. The somatic genomic landscape of glioblastoma. Cell. 2013; 155: 462-477.

7. Palumbo S, Miracco C, Pirtoli L, Comincini S. Emerging roles of microRNA in modulating cell-death processes in malignant glioma. J Cell Physiol. 2014; 229: 277-286.

8. Hermansen SK, Kristensen BW. MicroRNA biomarkers in glioblastoma. J Neurooncol. 2013; 114: 13-23.

9. Novakova J, Slaby O, Vyzula R, Michalek J. MicroRNA involvement in glioblastoma pathogenesis. Biochem Biophys Res Commun. 2009; 386: 1-5.

10. Memczak S, Jens M, Elefsinioti A, Torti F, Krueger J, Rybak A, Maier L, Mackowiak SD, Gregersen LH, Munschauer M, Loewer A, Ziebold U, Landthaler M et al. Circular RNAs are a large class of animal RNAs with regulatory potency. Nature. 2013; 495: 333-338.

11. Hansen TB, Jensen TI, Clausen BH, Bramsen JB, Finsen B, Damgaard CK, Kjems J. Natural RNA circles function as efficient microRNA sponges. Nature. 2013; 495: 384-388.

12. Hansen TB, Wiklund ED, Bramsen JB, Villadsen SB, Statham AL, Clark SJ, Kjems J. miRNA-dependent gene silencing involving Ago2-mediated cleavage of a circular antisense RNA. EMBO J. 2011; 30: 4414-4422.

13. Mermel CH, Schumacher SE, Hill B, Meyerson ML, Beroukhim R, Getz G. GISTIC2.0 facilitates sensitive and confident localization of the targets of focal somatic copynumber alteration in human cancers. Genome Biol. 2011; 12: R41.

14. Papp G, Krausz T, Stricker TP, Szendrői M, Sápi Z. SMARCB1 expression in epithelioid sarcoma is regulated by miR-206, miR-381, and miR-671-5p on both mRNA and protein levels. Genes Chromosomes Cancer. 2014; 53: $168-176$.

15. Rutnam ZJ, Yang BB. The non-coding 3' UTR of CD44 induces metastasis by regulating extracellular matrix functions. J Cell Sci. 2012; 125: 2075-2085.

16. Tarasov V, Jung P, Verdoodt B, Lodygin D, Epanchintsev A, Menssen A, Meister G, Hermeking H. Differential regulation of microRNAs by p 53 revealed by massively parallel sequencing: miR-34a is a p53 target that induces apoptosis and G1-arrest. Cell Cycle. 2007; 6: 1586-1593.

17. Li J, Yang J, Zhou P, Le Y, Zhou C, Wang S, Xu D, Lin HK, Gong Z. Circular RNAs in cancer: novel insights into origins, properties, functions and implications. Am J Cancer Res. 2015; 5: 472-480. 
18. Lukiw WJ. Circular RNA (circRNA) in Alzheimer's disease (AD). Front Genet. 2013; 4: 307.

19. Xu H, Guo S, Li W, Yu P. The circular RNA Cdr1as, via miR-7 and its targets, regulates insulin transcription and secretion in islet cells. Sci Rep. 2015; 5: 12453.

20. Chen YT, Rettig WJ, Yenamandra AK, Kozak CA, Chaganti RS, Posner JB, Old LJ. Cerebellar degenerationrelated antigen: a highly conserved neuroectodermal marker mapped to chromosomes X in human and mouse. Proc Natl Acad Sci U S A. 1990; 87: 3077-3081.

21. Fu J, Fong K, Bellacosa A, Ross E, Apostolou S, Bassi DE, Jin F, Zhang J, Cairns P, Ibañez de Caceres I, Braunewell $\mathrm{KH}$, Klein-Szanto AJ. VILIP-1 downregulation in nonsmall cell lung carcinomas: mechanisms and prediction of survival. PLoS One. 2008; 3: e1698.

22. Fu J, Jin F, Zhang J, Fong K, Bassi DE, Lopez De Cicco R, Ramaraju D, Braunewell KH, Conti C, Benavides F, KleinSzanto AJ. VILIP-1 expression in vivo results in decreased mouse skin keratinocyte proliferation and tumor development. PLoS One. 2010; 5: e10196.

23. Gonzalez Guerrico AM, Jaffer ZM, Page RE, Braunewell $\mathrm{KH}$, Chernoff J, Klein-Szanto AJ. Visinin-like protein-1 is a potent inhibitor of cell adhesion and migration in squamous carcinoma cells. Oncogene. 2005; 24: 2307-2316.

24. Yang G, Han D, Chen X, Zhang D, Wang L, Shi C, Zhang W, Li C, Chen X, Liu H, Zhang D, Kang J, Peng F et al. MiR-196a exerts its oncogenic effect in glioblastoma multiforme by inhibition of I $\mathrm{B} \alpha$ both in vitro and in vivo. Neuro Oncol. 2014; 16: 652-661.

25. Ma R, Yan W, Zhang G, Lv H, Liu Z, Fang F, Zhang W, Zhang J, Tao T, You Y, Jiang T, Kang X. Upregulation of miR-196b confers a poor prognosis in glioblastoma patients via inducing a proliferative phenotype. PLoS One. 2012; 7: e38096.

26. Lian S, Shi R, Bai T, Liu Y, Miao W, Wang H, Liu X, Fan Y. Anti-miRNA-23a oligonucleotide suppresses glioma cells growth by targeting apoptotic protease activating factor-1. Curr Pharm Des. 2013; 19: 6382-6389.

27. Chen L, Han L, Zhang K, Shi Z, Zhang J, Zhang A, Wang Y, Song Y, Li Y, Jiang T, Pu P, Jiang C, Kang C. VHL regulates the effects of miR-23b on glioma survival and invasion via suppression of HIF-1 $\alpha / \mathrm{VEGF}$ and $\beta$-catenin/ Tcf-4 signaling. Neuro Oncol. 2012; 14: 1026-1036.

28. Wang Z, Yang J, Xu G, Wang W, Liu C, Yang H, Yu Z, Lei Q, Xiao L, Xiong J, Zeng L, Xiang J, Ma J et al. Targeting miR-381-NEFL axis sensitizes glioblastoma cells to temozolomide by regulating stemness factors and multidrug resistance factors. Oncotarget. 2015; 6: 3147-3164. doi: 10.18632/oncotarget.3061.

29. Fang X, Yoon JG, Li L, Yu W, Shao J, Hua D, Zheng S, Hood L, Goodlett DR, Foltz G, Lin B. The SOX2 response program in glioblastoma multiforme: an integrated ChIPseq, expression microarray, and microRNA analysis. BMC Genomics. 2011; 12: 11 .
30. Berezovsky AD, Poisson LM, Cherba D, Webb CP, Transou AD, Lemke NW, Hong X, Hasselbach LA, Irtenkauf SM, Mikkelsen T, deCarvalho AC. Sox 2 promotes malignancy in glioblastoma by regulating plasticity and astrocytic differentiation. Neoplasia. 2014; 16: 193-206.

31. Hosseini Farahabadi SS, Ghaedi K, Ghazvini Zadegan F, Karbalaie K, Rabiee F, Nematollahi M, Baharvand H, Nasr-Esfahani MH. ERK1/2 is a key regulator of Fndc5 and PGC1 $\alpha$ expression during neural differentiation of mESCs. Neuroscience. 2015; 297: 252-261.

32. Ragusa M, Majorana A, Statello L, Maugeri M, Salito L, Barbagallo D, Guglielmino MR, Duro LR, Angelica R, Caltabiano R, Biondi A, Di Vita M, Privitera G et al. Specific alterations of microRNA transcriptome and global network structure in colorectal carcinoma after cetuximab treatment. Mol Cancer Ther. 2010; 9: 3396-3409.

33. Ragusa M, Majorana A, Banelli B, Barbagallo D, Statello L, Casciano I, Guglielmino MR, Duro LR, Scalia M, Magro G, Di Pietro C, Romani M, Purrello M. MIR152, MIR200B, and MIR338, human positional and functional neuroblastoma candidates, are involved in neuroblast differentiation and apoptosis. J Mol Med (Berl). 2010; 88: 1041-1053.

34. Caltabiano R, Barbagallo GM, Castaing M, Cassenti A, Senetta R, Cassoni P, Albanese V, Lanzafame S. Prognostic value of EGFR expression in de novo and progressed atypical and anaplastic meningiomas: an immunohistochemical and fluorescence in situ hybridization pilot study. J Neurosurg Sci. 2013; 57: 139-151.

35. Barbagallo GM, Paratore S, Caltabiano R, Palmucci S, Parra HS, Privitera G, Motta F, Lanzafame S, Scaglione G, Longo A, Albanese V, Certo F. Long-term therapy with temozolomide is a feasible option for newly diagnosed glioblastoma: a single-institution experience with as many as 101 temozolomide cycles. Neurosurg Focus. 2014; 37: E4.

36. Vandesompele J, De Preter K, Pattyn F, Poppe B, Van Roy N, De Paepe A, Speleman F. Accurate normalization of real-time quantitative RT-PCR data by geometric averaging of multiple internal control genes. Genome Biol. 2002; 3: RESEARCH0034.

37. Livak KJ, Schmittgen TD. Analysis of relative gene expression data using real-time quantitative PCR and the 2(-Delta Delta C(T)) Method. Methods. 2001; 25: 402-408.

38. Barbagallo D, Condorelli AG, Piro S, Parrinello N, Fløyel T, Ragusa M, Rabuazzo AM, Størling J, Purrello F, Di Pietro C, Purrello M. CEBPA exerts a specific and biologically important proapoptotic role in pancreatic $\beta$ cells through its downstream network targets. Mol Biol Cell. 2014; 25: 2333-2341.

39. Ragusa M, Statello L, Maugeri M, Majorana A, Barbagallo D, Salito L, Sammito M, Santonocito M, Angelica R, Cavallaro A, Scalia M, Caltabiano R, Privitera G et al. Specific alterations of the microRNA transcriptome and global network structure in colorectal cancer after treatment with MAPK/ ERK inhibitors. J Mol Med (Berl). 2012; 90: 1421-1438. 
40. Hsu SD, Lin FM, Wu WY, Liang C, Huang WC, Chan WL, Tsai WT, Chen GZ, Lee CJ, Chiu CM, Chien CH, Wu MC, Huang CY et al. miRTarBase: a database curates experimentally validated microRNA-target interactions. Nucleic Acids Res. 2011; 39: D163-169.

41. Lewis BP, Burge CB, Bartel DP. Conserved seed pairing, often flanked by adenosines, indicates that thousands of human genes are microRNA targets. Cell. 2005; 120: $15-20$.

42. Cho S, Jang I, Jun Y, Yoon S, Ko M, Kwon Y, Choi I, Chang H, Ryu D, Lee B, Kim VN, Kim W, Lee S. MiRGator v3.0: a microRNA portal for deep sequencing, expression profiling and mRNA targeting. Nucleic Acids Res. 2013; 41: D252-257.

43. Betel D, Koppal A, Agius P, Sander C, Leslie C. Comprehensive modeling of microRNA targets predicts functional non-conserved and non-canonical sites. Genome Biol. 2010; 11: R90.

44. Mostafavi S, Ray D, Warde-Farley D, Grouios C, Morris Q. GeneMANIA: a real-time multiple association network integration algorithm for predicting gene function. Genome Biol. 2008; 9: S4.

45. Warde-Farley D, Donaldson SL, Comes O, Zuberi K, Badrawi R, Chao P, Franz M, Grouios C, Kazi F, Lopes CT, Maitland A, Mostafavi S, Montojo J et al. The GeneMANIA prediction server: biological network integration for gene prioritization and predicting gene function. Nucleic Acids Res. 2010; 38: W214-220.

46. Su G, Morris JH, Demchak B, Bader GD. Biological network exploration with cytoscape 3. Curr Protoc Bioinformatics. 2014; 47: 8 .

47. Scardoni G, Petterlini M, Laudanna C. Analyzing biological network parameters with CentiScaPe. Bioinformatics. 2009; 25: 2857-2859.

48. Bindea G, Mlecnik B, Hackl H, Charoentong P, Tosolini M, Kirilovsky A, Fridman WH, Pagès F, Trajanoski Z, Galon J. ClueGO: a Cytoscape plug-in to decipher functionally grouped gene ontology and pathway annotation networks. Bioinformatics. 2009; 25: 1091-1093. 